\title{
Infliximab induces clinical, endoscopic and histological responses in refractory ulcerative colitis
}

\author{
F. Bermejo, A. López-Sanromán, J. Hinojosa ${ }^{1}$, L. Castro ${ }^{2}$, C. Jurado ${ }^{2}$ and A. B. Gómez-Belda ${ }^{1}$ \\ Gastroenterology Department. Ramón y Cajal University Hospital. Madrid. ${ }^{\prime}$ Digestive Diseases Section. Hospital de \\ Sagunto. Sagunto, Valencia. ${ }^{2}$ Digestive System Department. Virgen Macarena University Hospital. Seville. Spain
}

\begin{abstract}
Background: infliximab is a monoclonal antiTNF- $\alpha$ antibody that has repeatedly shown to be effective in the management of Crohn's disease. However, data are scarce about its efficacy in ulcerative colitis.

Aim: to describe the joint experience of three Spanish hospitals in the use of infliximab in patients with active refractory ulcerative colitis.

Patients and methods: we present seven cases of ulcerative colitis (6 with chronic active disease despite immunosuppressive therapy, and one with acute steroid-refractory ulcerative colitis) treated with infliximab $5 \mathrm{mg} / \mathrm{kg}$ of body weight. Clinical response was evaluated by means of the Clinical Activity Index at 2, 4 and 8 weeks after initial infusion. Biochemical (erythrocyte sedimentation rate and $\mathrm{C}$-reactive protein), endoscopic, and histological changes were also assessed.

Results: mean age of patients was $45.8 \pm 17$ years (range 23-77); 4 were female. No adverse effects were recorded. Inflammatory activity diminished significantly in 6 of 7 patients $(85.7 \%$; CI 95\%: 42-99\%) both from a clinical ( $\mathrm{p}=0.01)$ and biochemical ( $p<0.05)$ point of view. Five out of six patients $(83.3 \%$; 36-99\%) with corticosteroid-dependent disease could be successfully weaned off these drugs. Five patients were endoscopicly controlled both before and after therapy, and a positive endoscopic and histological response could be recorded in all of them.

Conclusion: infliximab may be an effective and safe therapy for some patients with ulcerative colitis refractory to other forms of therapy, although controlled studies are needed to assess its role in the general management of this disease.
\end{abstract}

Key words: Infliximab. Ulcerative colitis.

Bermejo F, López-Sanromán A, Hinojosa J, Castro L, Jurado $C$, Gómez-Belda AB. Infliximab induces clinical, endoscopic and histological responses in refractory ulcerative colitis. Rev Esp Enferm Dig 2004; 96: 94-101.

Recibido: 06-05-03.

Aceptado: 03-09-03.

Correspondencia: Fernando Bermejo San José. C/ Ríos Rosas, $17-5^{\circ} \mathrm{C}$. 28003 Madrid. Tel.: 9133992617. e-mail: fbermejos@medynet.com

\section{INTRODUCTION}

Tumor necrosis factor alpha (TNF- $\alpha$ ), a proinflammatory cytokine, plays a central role in the pathogenesis of chronic inflammatory bowel disease (IBD), especially in initiating and maintaining a pathological level of inflammation (1). Infliximab is a chimeric monoclonal $\mathrm{IgG}_{1}$ antibody directed against TNF- $\alpha$ that blocks both its soluble and transmembrane fractions; in addition, it neutralizes TNF- $\alpha$-synthesizing cells and induces apoptosis in activated $\mathrm{T}$ lymphocytes, which is down-regulated in patients with IBD (2). Infliximab has been shown to be efficacious and safe in the management of patients with both fistulous and luminal refractory Crohn's disease $(3,4)$, where its use is widespread $(5,6)$. Recent data suggest that it could also be of use in the management of ulcerative colitis (UC), although the number of studies and the amount of evaluated cases are still scant. Our aim was to describe the joint experience of three Spanish hospitals in the management of refractory UC with infliximab, and its effect on endoscopic and histological changes.

\section{METHODS}

Cases of patients with UC who were treated with infliximab in three Spanish hospitals were cross-sectionally analyzed. All had a definite diagnosis of UC according to widely accepted criteria $(7,8)$. The following data were gathered: epidemiological characterization, time since UC diagnosis, extension of colonic involvement, concomitant therapies when infliximab was administered, previous treatments failing to induce clinical remission and, eventually, adverse effects following infliximab administration.

Disease activity was graded according to the Clinical Activity Index (CAI), by Lichtiger et al (9), with a highest possible score of 21. To evaluate the success of infliximab treatment (significant diminution of CAI), this 
index was calculated before infusion and at 2, 4 and 8 weeks thereafter; the success of steroid weaning was also investigated in steroid-dependent patients (defined as complete withdrawal of corticosteroids in oral or topical administration) (10). In all cases, complete blood count, liver enzymes, erythrocyte sedimentation rate (ESR), and C-reactive protein were available before the infusion as well as 4 and 8 weeks thereafter. In all patients a colonoscopy with mucosal biopsies had been performed before infliximab therapy, and in 5 instances (all except numbers 6 and 7) a follow-up endoscopy with new biopsies was repeated at 6 to 8 weeks after infusion. To evaluate histological lesions, Riley's microscopic grading score was used (values from 0 to 4 ), a measure analyzing the degree of cellular inflammatory infiltrate and of tissue destruction present in the colonic mucosa (11).

Five of these patients were treated with a single IV infusion of infliximab, $5 \mathrm{mg} / \mathrm{kg}$ of body weight (Remicade ${ }^{\circledR}$, Centocor, Malvern, PA, U.S.A.) strictly adhering to the manufacturer's instructions, and following the regular legal procedure for off-label administration established by the Spanish Ministry of Health. In one of the hospitals (patients 6 and 7) a three-dose schedule was chosen $(0,2$ and 6 weeks), similar to what is widespread in the management of fistulous Crohn's disease refractory to immunosuppression $(4,5)$.

The statistical study was carried out using the SPSS10.0 pack. For qualitative variables, percentages and 95\% confidence intervals (CI 95\%) were calculated, and means and standard deviations were obtained for quantitative variables. A p value under 0.05 was considered statistically significant. An analysis of possible differences between pre- and post-treatment values was done by using Wilcoxon's test for paired data (signed rank test).

\section{RESULTS}

Seven patients were available for analysis: six cases of chronic active disease refractory to immunosuppressants and one case of acute severe colitis. In all of them baseline antinuclear antibodies were negative, as were the tests indicated to detect latent or active tuberculous infection (12). Prior to infusion, the diagnosis and the absence of cytomegalovirus superinfection were confirmed by endoscopy and biopsies in all cases. Mean age of patients was $45.8 \pm 17$ years (range 23-77); 4 were women. Clinical and demographic characteristics are defined in table I.

In case 1 , cyclosporine had proved ineffective, and case 2 had unsuccessfully been treated with cyclosporine and tacrolimus. No adverse effects were detected during infliximab administration. During follow-up, one patient with a previous diagnosis of extrinsic asthma was admitted for mild, acute respiratory failure 8 weeks after infliximab infusion, and then discharged uneventfully.

Inflammatory activity significantly diminished (treatment success) in six out of seven patients $(85.7 \%$; CI 95\%: 42-99\%) both from a clinical and biochemical point of view. In figure 1, we depict the evolution of CAI, which significantly dropped two weeks after infusion ( $p$ $=0.01$ ), a tendency kept for weeks 4 and 8. Erythrocyte sedimentation rate and $\mathrm{C}$-reactive protein values also descended significantly $(\mathrm{p}<0.05)$ at week 4 (Table II).

In five out of six patients with steroid-dependent disease, these drugs could be successfully withdrawn (83.3\%; 36-99\%). In cases 1 and 2 (6 months follow-up) and in case 3 (3 months follow-up), response was successfully maintained by infliximab infusions $(5 \mathrm{mg} / \mathrm{kg})$ every 8 weeks. Patients are treated with azathioprine and remain clinically asymptomatic (IAC: 0-2) and with normal values of C-reactive protein. In cases 4, 5 and 6, no maintenance infusions were administered, and all are still in clini-

Table I. Clinical and demographic characteristics of patients

\begin{tabular}{|c|c|c|c|c|c|c|c|}
\hline & Case 1 & Case 2 & Case 3 & Case 4 & Case 5 & Case 6 & Case 7 \\
\hline Age & 55 & 31 & 77 & 23 & 37 & 45 & 53 \\
\hline Gender & Male & Male & Female & Female & Male & Female & Female \\
\hline Tobacco & Ex-smoker & Never & Never & Never & Never & Never & Never \\
\hline Years of disease & 10 & 4 & 1 & 5,5 & 5 & 6 & 10 \\
\hline ANCA & - & + & + & - & - & - & - \\
\hline $\begin{array}{l}\text { Concomitante } \\
\text { therapy } \\
\text { (PO daily dose) }\end{array}$ & $\begin{array}{l}\text { PDN } 20 \mathrm{mg} \\
\text { AZA } 150 \mathrm{mg} \\
\text { MSZ } 3 \mathrm{~g}\end{array}$ & $\begin{array}{l}\text { PDL } 40 \mathrm{mg} \\
\text { AZA } 150 \mathrm{mg}\end{array}$ & $\begin{array}{l}\text { PDL } 40 \mathrm{mg} \\
\text { MP } 75 \mathrm{mg}\end{array}$ & $\begin{array}{l}\text { PDN } 40 \mathrm{mg} \\
\text { AZA } 100 \mathrm{mg} \\
\text { MSZ } 2 \mathrm{~g} \\
\text { BUD enema }\end{array}$ & $\begin{array}{l}\text { PDN } 30 \mathrm{mg} \\
\text { AZA } 125 \mathrm{mg} \\
\text { MSZ } 2 \mathrm{~g}\end{array}$ & $\begin{array}{l}\text { PDN } 30 \mathrm{mg} \\
\text { AZA } 150 \mathrm{mg}\end{array}$ & $\begin{array}{l}\text { PDN } 40 \mathrm{mg} \\
\text { AZA } 150 \mathrm{mg}\end{array}$ \\
\hline Corticosteroids & Dependency & Dependency & Dependency & Dependency & Refractory & Dependency & Dependency \\
\hline Extension & Left sided colitis & Pancolitis & Left sided colitis & Pancolitis & Proctosigmoiditis & Left sided colitis & Proctosigmoiditis \\
\hline $\begin{array}{l}\text { Extraintestinal } \\
\text { manifestations }\end{array}$ & None & None & None & $\begin{array}{l}\text { Erythema nodosum } \\
\text { Arthralgia }\end{array}$ & $\begin{array}{l}\text { Spondilytis } \\
\text { HLA-B27 + }\end{array}$ & Arthralgias & Artrralgias \\
\hline
\end{tabular}

PDN: prednisone. PDL: prednisolone, AZA: azathioprine. MP: mercaptopurine. MSZ: mesalamine. BUD: budesonide. vo: per os. 


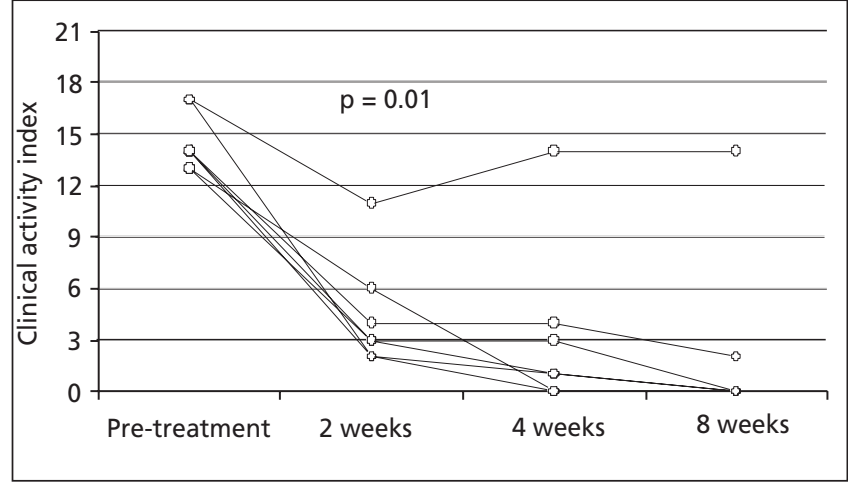

Fig. 1. Clinical activity index before and after infliximab treatment.

cal remission (6 months follow-up). The seventh patient still suffers from chronic active disease.

Endoscopic follow-up is available for 5 patients, all of them clinical responders. Endoscopic lesions are significantly milder, which was confirmed by biopsies: in all cases, post-treatment Riley's score is significantly lower (Table III).

\section{DISCUSSION}

TNF- $\alpha$ is a key cytokine in the pathogenesis of IBD. It plays a leading role in inflammatory processes, with an im- portant contribution to macrophage and neutrophil activation, increase of capillary permeability, extrinsic activation of coagulation pathways, and recruitment of various immunologic cells (13). Its role in the inhibition of T-lymphocyte apoptosis is also very striking. Classically, Crohn's disease and UC are described as corresponding to a Th1 (proinflammatory, TNF- $\alpha$-mediated) and a Th2 (humoral) response, respectively (14). However, there is enough scientific evidence pointing at the important role played by TNF- $\alpha$ in UC (13), which supports the possible usefulness of antiTNF- $\alpha$ therapy in this disease. Different authors have described active TNF- $\alpha$ synthesis both locally and systemically, as shown by an increased production in the colonic mucosa (15-17), and an increased detection in the stools, plasma, urine and rectal dyalisates of patients with active UC (1820). Moreover, this increase correlates with disease severity as measured by clinical or laboratory parameters. A second line of evidence is supplied by the experimental treatment of animals with idiopathic UC using anti-TNF- $\alpha$ antibodies (CDP571). Such treatment is followed by weight gain and a partial reversal of histological mucosal changes (21).

Clinical situations where infliximab could be useful in UC include severe acute steroid-refractory disease, and chronic active disease refractory to immunosuppressants (azathioprine or 6-mercaptopurine). In the first case, infliximab could be an alternative to cyclosporine, with the additional advantages of easier administration and no need to monitor blood levels (which makes the use of cyclosporine

Table II. Inflammatory markers before and after infliximab treatment

\begin{tabular}{lcccccc}
\hline & \multicolumn{3}{c}{ ERS $(\mathrm{mm} / \mathrm{h})$} & \multicolumn{3}{c}{ CRP $(\mathrm{mg} / \mathrm{l})$} \\
\hline Case 1 & Baseline & 4 weeks & 8 weeks & Baseline & 4 weeks & 8 weeks \\
Case 2 & 74 & 25 & 13 & 70 & 3 & 3 \\
Case 3 & 91 & 11 & 12 & 52 & 9 & 4 \\
Case 4 & 72 & 31 & 46 & 15 & 3 & 3 \\
Case 5 & 33 & 8 & 8 & 16 & 3 & 3 \\
Case 6 & 68 & 40 & 2 & 35 & 3 & 3 \\
Case 7 & 22 & 10 & 6 & 8 & 3 & 3 \\
\hline
\end{tabular}

ESR: erythrocite sedimentation rate. CRP: C-reactive protein

Table III. Endoscopic and histologic findings before and after infliximab therapy

\begin{tabular}{lcccc}
\hline & \multicolumn{2}{c}{ Endoscopic activity } & \multicolumn{2}{c}{ Histologic activity (Riley) } \\
\hline Case 1 & Pre-treatment & Post-treatment & Pre-treatment & Post-treatment \\
Case 2 & Severe & Mild & 4 & 1 \\
Case 3 & Severe & Mild & 4 & 1 \\
Case 4 & Severe & Mild & 4 & 2 \\
Case 5 & Severe & Inactive & 3 & 1 \\
Case 6 & Severe & Inactive & 4 & 1 \\
Case 7 & Moderate & Not available & 3 & Not available \\
\hline
\end{tabular}


difficult in some settings). In such patients, data about infliximab efficacy are contradictory, with response rates varying between 33 and 100\% (22-30). A recent placebo-controlled trial does not support the use of infliximab in this group of patients (29). Only one of our patients was treated for acute steroid-refractory colitis, with optimal results.

Another possible application is chronically active colitis that is refractory to immunosuppressive treatment, a situation where evidence is still more scarce and results very uneven, with response rates ranging from $0 \%$ (31) to $90 \%$ (25). In our series this indication was present in six patients, five of which showed a favourable response $(83 \%)$ in accordance with observations by Su et al. (25). In our steroid-dependent patients those drugs were successfully withdrawn, as described by these authors (25).

The clinical response observed in a majority $(85 \%)$ of our patients was supported by a significant descent in inflammatory markers. Endoscopic severity diminished significantly as well, and so did histological activity.

Finally, our results suggest that infliximab could be an alternative when other forms of treatment have failed. It is important to point out that, according to classical management, all our patients would have been candidates to colectomy, a procedure not free of morbidity and mortality (32). In spite of those promising results, it is important to be aware that the size of published series is small, severity of patients is not homogeneous, and doses and schedules vary. All this points to a need for multicenter, controlled, randomized studies to establish the true role infliximab may play for patients with UC mainly in two clinical situations: steroid-refractory disease, and chronic active colitis refractory to immunosuppressants.

\section{REFERENCES}

1. Hinojosa J. Anticuerpos anti-TNF en el tratamiento de la enfermedad inflamatoria intestinal. Gastroenterol Hepatol 2000; 23: 250-7.

2. Sandborn WJ, Targan SR. Biologic therapy of inflammatory bowel disease. Gastroenterol 2002; 122: 1592-608.

3. Targan SR, Hanauer SB, van Deventer SJ, Mayer L, Present DH, Braakman T, et al. A short-term study of chimeric monoclonal antibody cA2 to tumor necrosis factor alpha for Crohn's disease. Crohn's Disease cA2 Study Group. N Engl J Med 1997; 337: 1029-35.

4. Present DH, Rutgeerts P, Targan SR, Hanauer SB, Mayer L, van Hogezand RA, et al. Infliximab for the treatment of fistulas in patients with Crohn's disease. N Engl J Med 1999; 340: 1398-405.

5. Domenech E, Esteve-Comas M, Gomollón F, Hinojosa J, Obrador A, Panés J, et al. Recomendaciones para el uso de infliximab (Remicade®) en la enfermedad de Crohn. GETECCU 2001. Gastroenterol Hepatol 2002; 25: 162-8.

6. Caprilli R, Viscido A, Guagnozzi D. Review article: biological agents in the treatment of Crohn's disease. Aliment Pharmacol Ther 2002; 16: $1579-90$

7. Lennard-Jones JE. Classification of inflammatory bowel disease. Scand J Gastroenterol 1989; 170: S2-S6.

8. O’Morain C, Tobin A, Leen E, Suzuki Y, O'Riordan T. Criteria of case definition in Crohn's disease and ulcerative colitis. Scand J Gastroenterol 1989; 170: S7-S11.

9. Lichtiger SC, Present DH, Kornbluth A, Gelernt I, Bauer J, Galler G, et al. Cyclosporin in severe ulcerative colitis refractory to steroid therapy. N Engl J Med 1994; 330: 1841-5.
10. Faubion WA, Loftus EV, Harmsen WS, Zinsmeister AR, Sandborn WJ. The natural history of corticosteroid therapy for inflammatory bowel disease: a population-based study. Gastroenterology 2001; 121: 255-60.

11. Riley SA, Mani V, Goodman MJ, Herd ME, Dutt S. Comparison of delayed release 5 aminosalicylic acid (mesalazine) and sulphasalazine in the treatment of mild to moderate ulcerative colitis relapse. Gut 1988; 29: 669-74.

12. Obrador A, López San Román A, Muñoz P, Fortun J, Gassull MA. Guía de consenso sobre tuberculosis y tratamiento de la enfermedad inflamatoria intestinal con infliximab. Gastroenterol Hepatol 2003; 26: 29-33.

13. Lichtenstein GR. Is Infliximab effective for induction of remission in patients with ulcerative colitis? Infl Bowel Dis 2001; 7: 89-93.

14. Fiocchi C. Inflammatory bowel disease: etiology and pathogenesis. Gastroenterology 1998; 115: 182-205.

15. Reinecker HC, Steffen M, Witthoeft T, Pflueger I, Schreiber S, MacDermott RP, et al. Enhanced secretion of tumour necrosis factor-alpha, IL-6, and IL-1 beta by isolated lamina propria mononuclear cells from patients with ulcerative colitis and Crohn's disease. Clin Exp Immunol 1993; 94: 174-81.

16. Masuda H, Iwai S, Tanaka T, Hayakawa S. Expression of IL-8, TNFalpha and IFN-gamma m-RNA in ulcerative colitis, particularly in patients with inactive phase. J Clin Lab Immunol 1995; 46: 111-23.

17. Reimund JM, Wittersheim C, Dumont S, Muller CD, Baumann R, Poindron $\mathrm{P}$, et al. Mucosal inflammatory cytokine production by intestinal biopsies in patients with ulcerative colitis and Crohn's disease. J Clin Immunol 1996; 16: 144-50.

18. Nielsen OH, Gionchetti P, Ainsworth M, Vainer B, Campieri M, Borregaard N, et al. Rectal dialysate and fecal concentrations of neutrophil gelatinase-associated lipocalin, interleukin-8, and tumor necrosis factoralpha in ulcerative colitis. Am J Gastroenterol 1999; 94: 2923-8.

19. Saiki T, Mitsuyama K, Toyonaga A, Ishida H, Tanikawa K. Detection of pro- and anti-inflammatory cytokines in stools of patients with inflammatory bowel disease. Scand J Gastroenterol 1998; 33: 616-22.

20. Hadziselimovic F, Emmons LR, Gallati H. Soluble tumour necrosis factor receptors p55 and p75 in the urine monitor disease activity and the efficacy of treatment of inflammatory bowel disease. Gut 1995; 37: 260-3.

21. Evans RC, Clarke L, Heath P, Stephens S, Morris AI, Rhodes JM. Treatment of ulcerative colitis with an engineered anti-TNF alpha antibody CDP 571. Aliment Pharmacol Ther 1997; 11: 1031-5.

22. Sands BE, Tremaine WJ, Sandborn WJ, Rutgeerts PJ, Hanauer SB, Mayer L, et al. Infliximab in the treatment of severe steroid-refractory ulcerative colitis: A pilot study. Infl Bowel Dis 2001; 7: 83-8.

23. Chey WY, Hussain A, Ryan C, Potter GD, Shah A. Infliximab for refractory ulcerative colitis. Am J Gastroentrol 2001; 96: 2373-81.

24. Kaser A, Mairinger T, Vogel W, Tilg H. Infliximab in severe steroidrefractory ulcerative colitis: A pilot study. Wien Klin Wochenschr 2001; 113: 930-3.

25. Su C, Salzberg BA, Lewis JD, Deren J, Kornbluth A, Katzka DA, et al. Efficacy of anti-tumor necrosis factor therapy in patients with ulcerative colitis. Am J Gastroenterol 2002; 97: 2576-84.

26. Kohn A, Prantera C, Pera A, Cosintino R, Sostegni R, Daperno M. Anti-tumour necrosis factor alpha (infliximab) in the treatment of severe ulcerative colitis: result of an open study on 13 patients. Dig Liver Dis 2002; 34: 626-30.

27. Actis GC, Bruno M, Pinna-Pintor M, Rossini FP, Rizzetto M. Infliximab for treatment of steroid-refractory ulcerative colitis. Dig Liver Dis 2002; 34: 631-4.

28. Castro Fernández M, García Díaz E, Romero M, Galán Jurado V, Rodríguez Alonso C. Tratamiento de la colitis ulcerosa corticorrefractaria con infliximab. Gastroenterol Hepatol 2003; 26: 54-5.

29. Probert CSJ, Hearing SD, Schreiber S, Kühbacher T, Ghosh S, Arnott IDR, et al. Infliximab in moderately severe glucocorticoid resistant ulcerative colitis: a randomised controlled trial. Gut 2003; 52: 998-1002.

30. Gornet JM, Couve S, Hassani Z, Delchier JC, Marteau P, Cosnes J, et al. Infliximab for refractory ulcerative colitis or indeterminate colitis: an open-label multicentre study. Aliment Pharmacol Ther 2003; 18: 175-81.

31. Kugathasan S, Prajapati DN, Kim JP, Saeian K, Emmons J, Knox J, et al. Infliximab outcome in children and adults with ulcerative colitis. Gastroenterology 2002; 122: A-615.

32. Binderow SR, Wexner SD. Current surgical therapy for mucosal ulcerative colitis. Dis Colon Rectum 1994; 37: 610-24. 


\title{
Infliximab induce respuesta clínica, endoscópica e histológica en la colitis ulcerosa refractaria
}

\author{
F. Bermejo, A. López-Sanromán, J. Hinojosa ${ }^{1}$, L. Castro 2 , C. Jurado ${ }^{2}$ y A. B. Gómez-Belda ${ }^{1}$ \\ Servicio de Gastroenterología. Hospital Ramón y Cajal. Madrid. 'Sección de Digestivo. Hospital de Sagunto. Sagunto, \\ Valencia. ${ }^{2}$ Servicio de Aparato Digestivo. Hospital Virgen de Macarena. Sevilla
}

\section{RESUMEN}

Introducción: infliximab, un anticuerpo monoclonal quimérico antiTNF- $\alpha$ ha demostrado su eficacia en pacientes con enfermedad de Crohn. Sin embargo, son escasos los datos sobre su efectividad en el tratamiento de la colitis ulcerosa.

Objetivo: describir la experiencia conjunta de 3 hospitales españoles en el uso de infliximab en enfermos con $\mathrm{CU}$ activa resistente a otros tratamientos.

Pacientes y métodos: se presentan 7 casos de colitis ulcerosa 6 con enfermedad crónicamente activa a pesar de tratamiento con inmunosupresor y 1 con colitis aguda grave refractaria a esteroides) tratados con infliximab a dosis de $5 \mathrm{mg} / \mathrm{kg}$ de peso. Se evaluó la respuesta clínica mediante un Índice de Actividad Clínica trascurridas 2, 4 y 8 semanas de la infusión inicial. Así mismo, se estudiaron los cambios analíticos (velocidad de sedimentación y proteína $\mathrm{C}$ reactiva), endoscópicos e histológicos.

Resultados: la edad media de los enfermos fue de $45,8 \pm 17$ años (rango 23-77); 4 de ellos eran mujeres. La actividad inflamatoria disminuyó significativamente en 6 de los 7 pacientes (85,7\%; IC95\%: 42-99\%) tanto desde el punto de vista clínico $(p=0,01)$ como analítico $(p<0,05)$. En 5 de los 6 pacientes con criterios de dependencia a esteroides se consiguió la retirada de los mismos (83,3\%; 36-99\%). Se obtuvo la mejoría de las lesiones endoscópicas e histológicas en los 5 enfermos en los que se había realizado endoscopia antes y después del tratamiento.

Conclusión: infliximab puede ser una terapia eficaz y segura en algunos enfermos con colitis ulcerosa resistente a otras alternativas terapéuticas, aunque se precisan estudios controlados para conocer su papel en el tratamiento de esta enfermedad.

Palabras clave: Infliximab. Colitis ulcerosa.

\section{INTRODUCCIÓN}

El factor de necrosis tumoral alfa (TNF- $\alpha$ ) es una citocina proinflamatoria clave en la patogenia de la enfermedad inflamatoria crónica intestinal (EICI), especialmente en el inicio y mantenimiento del proceso patológico (1). Infliximab (IFX) es un anticuerpo monoclonal quimérico antiTNF- $\alpha$ de tipo $\operatorname{IgG}_{1}$, cuyo mecanismo de acción incluye la neutralización de la parte soluble y de la porción transmembrana del TNF- $\alpha$; además, neutraliza las células productoras de TNF- $\alpha$ e induce la apoptosis de linfocitos
T activados, que en pacientes con EICI se encuentra inhibida (2). Infliximab ha demostrado su eficacia en el tratamiento de pacientes con enfermedad de Crohn fistulosa e inflamatoria refractaria $(3,4)$, en la que actualmente está indicado su uso $(5,6)$. Recientes datos sugieren que también podría ser efectivo en el tratamiento de la colitis ulcerosa (CU), aunque el número de estudios y la cantidad de pacientes evaluados son aún escasos. El objetivo de este trabajo fue describir la experiencia conjunta de tres hospitales españoles en el uso de IFX en enfermos con CU resistente al tratamiento habitual y el efecto del fármaco en los cambios endoscópicos e histológicos.

\section{MÉTODOS}

Se realizó un estudio transversal investigando los pacientes diagnosticados de CU que habían recibido IFX en tres hospitales españoles. Todos los enfermos habían sido diagnosticados de CU definitiva mediante los criterios habituales $(7,8)$. Se recogieron los datos epidemiológicos de los enfermos, el tiempo de evolución de la enfermedad desde el diagnóstico, la extensión de la afectación colónica, el tratamiento concomitante en el momento de la administración de IFX, los tratamientos que habían fracasado previamente en inducir la remisión clínica y los eventuales efectos adversos tras la administración de IFX.

La actividad de la enfermedad se estableció mediante el Índice de Actividad Clínica (IAC) de Lichtiger y cols. (9), cuya puntuación máxima es de 21. Para evaluar el éxito del tratamiento con IFX (descenso significativo del IAC) se calculó el IAC al inicio y transcurridas 2,4 y 8 semanas del tratamiento, y se recogieron los datos referentes a la consecución o no de la retirada de los esteroides (suspensión completa de estos fármacos en administración oral o tópica) en los pacientes dependientes de esteroides (10). Asimismo, se recogieron en todos los pacientes los datos de hemograma, bioquímica hepática, velocidad de sedimentación y proteína $\mathrm{C}$ reactiva, antes del tratamiento y trascurridas 4 y 8 semanas del mismo. Ade- 
más, en todos los casos se había practicado una endoscopia con biopsias antes del tratamiento, y 6-8 semanas después del mismo se realizó un control endoscópico en 5 pacientes (todos excepto los casos 6 y 7). Para evaluar las lesiones histológicas se había utilizado la escala de gradación microscópica de Riley (desde 0 hasta 4) que valora el grado de infiltrado inflamatorio celular y de destrucción tisular presentes en la mucosa del colon (11).

Cinco de los pacientes recibieron una infusión de $5 \mathrm{mg} / \mathrm{kg}$ de peso de infliximab (Remicade ${ }^{\circledR}$, Centocor, Malvern, PA, USA) siguiendo las normas de administración que indica la ficha técnica y previa autorización de uso compasivo por el Ministerio de Sanidad y Consumo. En uno de los centros (casos 6 y 7) se optó por una pauta de administración de 3 infusiones (inicial, a las 2 y 6 semanas) similar a la descrita en la enfermedad de Crohn fistulosa sin respuesta a tratamiento antibiótico e inmunosupresor $(4,5)$.

Para el estudio estadístico se utilizó el programa SPSS 10.0. En las variables cualitativas se calcularon el porcentaje y su intervalo de confianza al 95\% (IC 95\%), y en las cuantitativas, la media aritmética y su desviación estándar. Consideramos estadísticamente significativo un valor de $\mathrm{p}<0,05$. El análisis de las diferencias entre los resultados previos y posteriores al tratamiento se realizó mediante el test de Wilcoxon para datos apareados (signed rank test).

\section{RESULTADOS}

Se recogieron los datos correspondientes a 7 pacientes: 6 de ellos con enfermedad crónicamente activa a pesar de tratamiento inmunosupresor y uno con colitis aguda grave. En todos ellos, la determinación basal de anticuerpos antinucleares fue negativa, así como los estudios encaminados a detectar infección tuberculosa activa o latente (12). Se descartó la existencia de superinfección por citomegalovirus mediante endoscopia y biopsias antes de la infusión en todos los casos. La edad media de los enfermos fue de 45,8 \pm 17 años (rango 23-77); 4 de ellos eran mujeres. Las características clínico-demográficas de los pacientes se definen en la tabla I. En el caso 1 había fracasado la administración de ciclosporina y en el caso 2 había fracasado el tratamiento tanto con ciclosporina como con tacrolimus. No se objetivaron efectos secundarios en ningún caso durante la administración de IFX. En el seguimiento, una paciente previamente diagnosticada de asma extrínseca, sufrió un episodio de reagudización de la misma a las 8 semanas de la infusión de IFX, y fue dada de alta sin complicaciones.

La actividad inflamatoria disminuyó significativamente (éxitos del tratamiento) en 6 de los 7 pacientes $(85,7 \%$; IC95\%: 42-99\%) tanto desde el punto de vista clínico como analítico. En la figura 1 se describe la evolución del IAC, que descendió significativamente a las 2 semanas de la infusión ( $\mathrm{p}=0,01)$, manteniéndose el descenso en las semanas 4 y 8 . Los valores de la velocidad de sedimentación y la proteína $\mathrm{C}$ reactiva disminuyeron significativamente ( $\mathrm{p}<0,05)$ a las 4 semanas (Tabla II).

En 5 de los 6 pacientes con criterios de dependencia a esteroides se consiguió la retirada de los mismos $(83,3 \%$; $36-$ $99 \%$ ). En los casos 1 y 2 (seguimiento de 6 meses) y en el caso 3 (seguimiento 3 meses) la respuesta ha sido mantenida con éxito mediante infusiones de IFX $(5 \mathrm{mg} / \mathrm{kg})$ cada 8 semanas. Los enfermos están siendo tratados con azatioprina, clínicamente asintomáticos (IAC: 0-2) y con proteína C reactiva en valores normales. En los casos 4, 5 y 6 no se realizaron nuevas infusiones y se encuentran actualmente en remisión clínica (seguimiento 6 meses). El caso 7 mantiene la enfermedad crónicamente activa.

Se dispone de seguimiento endoscópico en 5 pacientes, todos ellos respondedores. Las lesiones endoscópicas eran significativamente más leves, lo que se confirmó mediante las biopsias: en todos los casos el índice de Riley postratamiento fue significativamente más bajo (Tabla III).

Tabla I. Características clínico-demográficas de los pacientes

\begin{tabular}{|c|c|c|c|c|c|c|c|}
\hline & Caso 1 & Caso 2 & Caso 3 & Caso 4 & Caso 5 & Caso 6 & Caso 7 \\
\hline Edad & 55 & 31 & 77 & 23 & 37 & 45 & 53 \\
\hline Sexo & Varón & Varón & Mujer & Mujer & Varón & Mujer & Mujer \\
\hline Tabaco & Exfumador & No & No & No & No & No & No \\
\hline Años de evolución & 10 & 4 & 1 & 5,5 & 5 & 6 & 10 \\
\hline ANCA & - & + & + & - & - & - & - \\
\hline $\begin{array}{l}\text { Terapia } \\
\text { concomitante } \\
\text { (dosis v.o. / } 24 \text { h) }\end{array}$ & $\begin{array}{l}\text { PDN } 20 \mathrm{mg} \\
\text { AZA } 150 \mathrm{mg} \\
\text { MSZ } 3 \mathrm{~g}\end{array}$ & $\begin{array}{l}\text { PDL } 40 \mathrm{mg} \\
\text { AZA } 150 \mathrm{mg}\end{array}$ & $\begin{array}{l}\text { PDL } 40 \mathrm{mg} \\
\text { MP } 75 \mathrm{mg}\end{array}$ & $\begin{array}{l}\text { PDN } 40 \mathrm{mg} \\
\text { AZA } 100 \mathrm{mg} \\
\text { MSZ } 2 \mathrm{~g} \\
\text { BUD enema }\end{array}$ & $\begin{array}{l}\text { PDN } 30 \mathrm{mg} \\
\text { AZA } 125 \mathrm{mg} \\
\text { MSZ } 2 \mathrm{~g}\end{array}$ & $\begin{array}{l}\text { PDN } 30 \mathrm{mg} \\
\text { AZA } 150 \mathrm{mg}\end{array}$ & $\begin{array}{l}\text { PDN } 40 \mathrm{mg} \\
\text { AZA } 150 \mathrm{mg}\end{array}$ \\
\hline Esteroides & Dependencia & Dependencia & Dependencia & Dependencia & Refractaria & Dependencia & Dependencia \\
\hline Extensión & Colitis izquierda & Pancolitis & Colitis izquierda & Pancolitis & Proctosigmoiditis & Colitis izquierda & Proctosigmoiditis \\
\hline $\begin{array}{l}\text { Manifestaciones } \\
\text { extraintestinales }\end{array}$ & No & No & No & $\begin{array}{l}\text { Eritema nodoso } \\
\text { Artralgias }\end{array}$ & $\begin{array}{l}\text { Espondilitis } \\
\text { HLA-B27 + }\end{array}$ & Artralgias & Artralgias \\
\hline
\end{tabular}

PDN: prednisona. PDL: prednisolona, AZA: azatioprina. MP: mercaptopurina. MSZ: mesalazina. BUD: budesonida. v.o.: vía oral. 


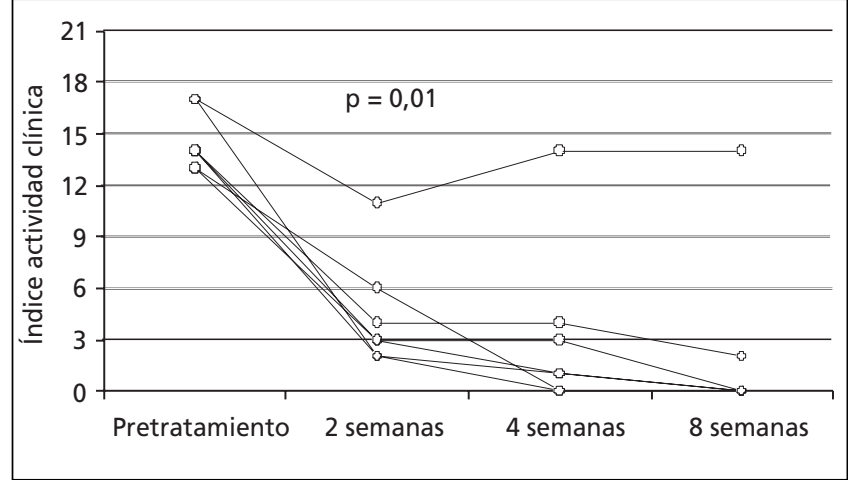

Fig. 1. Indice de actividad clínica antes y después del tratamiento con infliximab.

\section{DISCUSIÓN}

El TNF- $\alpha$ es una citocina clave en la patogenia de la EICI. Posee un papel primordial en la cascada inflamatoria contribuyendo a la activación de macrófagos y neutrófilos, al aumento de la permeabilidad capilar, a la activación de la vía extrínseca de la coagulación y al reclutamiento de diferentes células inmunológicas (13). También parece desempeñar un papel en la inhibición de la apoptosis de los linfocitos T. Clásicamente se describe a la enfermedad de Crohn como una entidad mediada por una respuesta Th1 (proinflamatoria, mediada por TNF- $\alpha$ ), mientras que en la CU la respuesta sería de tipo Th2 (humoral) (14). Sin embargo, existe evidencia científica que confirma el papel importante del TNF- $\alpha$ en la CU (13), lo que apoya la posible utilidad de la terapia anti-TNF en el tratamiento de esta entidad clínica. Diversos autores han descrito altas tasas de producción local y sistémica del TNF, que se demuestra por el aumento de la producción de esta citocina en la mucosa colónica (15-17), en las heces, plasma, orina o dializado rectal de los enfermos con CU activa (18-20). Además, este aumento de concentración se correlaciona con la gravedad de la enfermedad, tanto clínica como analítica. La segunda línea de evidencia proviene del tratamiento de animales de experimentación con CU idiopática con anticuerpos anti-TNF (CDP571). Dicho tratamiento conduce a aumento de peso y una mejoría histológica de la inflamación mucosa (21).

Las situaciones clínicas en las que infliximab podría ser útil en la CU serían la enfermedad aguda grave refractaria a esteroides, y la CU crónicamente activa a pesar del tratamiento con inmunosupresores (azatioprina o mercaptopurina). En la primera situación, infliximab podría constituir una alternativa a la ciclosporina con la ventaja de una administración más sencilla que no precisa la monitorización de niveles del fármaco (lo que imposibilita el uso de ciclosporina en algunos centros). En este tipo de enfermos los datos de eficacia de infliximab son contradictorios con porcentajes de respuesta descritos que oscilan entre el 33 y el 100\% (22-30). Un reciente estudio

Tabla II. Valores de los marcadores de inflamación previos y posteriores al tratamiento con infliximab

\begin{tabular}{|c|c|c|c|c|c|c|}
\hline & \multicolumn{3}{|c|}{$V S G(m m / h)$} & \multicolumn{3}{|c|}{$P C R(m g / l)$} \\
\hline & Inicial & 4 semanas & 8 semanas & Inicial & 4 semanas & 8 semanas \\
\hline Caso 1 & 74 & 25 & 13 & 70 & 3 & 3 \\
\hline Caso 2 & 91 & 11 & 12 & 52 & 9 & 4 \\
\hline Caso 3 & 72 & 31 & 46 & 15 & 3 & 3 \\
\hline Caso 4 & 33 & 8 & 8 & 16 & 3 & 3 \\
\hline Caso 5 & 68 & 40 & 2 & 35 & 3 & 3 \\
\hline Caso 6 & 22 & 10 & 6 & 8 & 3 & 3 \\
\hline Caso 7 & 17 & 31 & 60 & 10 & 9 & 18 \\
\hline
\end{tabular}

VSG: velocidad de sedimentación globular. PCR: proteína C reactiva

Tabla III. Hallazgos endoscópicos e histológicos previos y posteriores al tratamiento

\begin{tabular}{lcccc}
\hline & \multicolumn{2}{c}{ Actividad endoscópica } & \multicolumn{2}{c}{ Actividad histológica (Riley) } \\
\hline Caso 1 & Pretratamiento & Postratamiento & Pretratamiento & Postratamiento \\
Caso 2 & Grave & Leve & 4 & 1 \\
Caso 3 & Grave & Leve & 4 & 1 \\
Caso 4 & Grave & Leve & 4 & 2 \\
Caso 5 & Grave & Inactiva & 3 & 1 \\
Caso 6 & Grave & Inactiva & 4 & 1 \\
Caso 7 & Moderada & No disponible & 3 & No disponible \\
\hline
\end{tabular}


controlado con placebo no apoya el uso de infliximab en este grupo de pacientes (29). Sólo uno de nuestros enfermos recibió infliximab por situación clínica de colitis refractaria a esteroides y el resultado fue óptimo.

Otra posible aplicación es la colitis crónicamente activa a pesar de tratamiento inmunosupresor, situación en la que existen menos datos publicados, y los resultados son dispares, con porcentajes de respuesta que oscilan entre el $0 \%$ (31) y $90 \%$ (25). En nuestra serie esta fue la indicación de tratamiento en 6 casos, 5 de los cuales respondieron favorablemente (83\%), lo que concuerda con los datos observados por $\mathrm{Su}$ y cols. (25). Como han descrito estos autores, en todos nuestros pacientes con criterios de dependencia a esteroides se consiguió la retirada de los mismos (25).

La mejoría clínica observada en la mayoría (85\%) de los enfermos fue corroborada por el descenso significativo en los parámetros analíticos de inflamación. La grave- dad de las lesiones endoscópicas y de la actividad histológica también disminuyeron significativamente.

Por último, nuestros resultados sugieren que infliximab podría ser una alternativa cuando han fracasado otras formas de tratamiento. Es necesario tener en cuenta, que, de haber seguido las pautas de tratamiento clásicas, todos nuestros pacientes hubieran sido candidatos a colectomía, un procedimiento no exento de morbimortalidad (32). A pesar de los prometedores resultados, es preciso ser conscientes de que el tamaño de las series publicadas es pequeño, la gravedad de los enfermos no es homogénea, y las dosis y los esquemas de tratamiento varían. Todo ello nos indica la necesidad de ensayos multicéntricos, controlados y aleatorizados que puedan delimitar el verdadero papel de infliximab en los pacientes con $\mathrm{CU}$, principalmente en dos situaciones clínicas de esta entidad: enfermedad refractaria a esteroides y colitis crónica activa a pesar de tratamiento inmunosupresor. 